\title{
Mercado de trabajo rural y precarización: nuevas condiciones socioeconómicas en el sur del Estado de México
}

\author{
Elizabeth Guadalupe Chong González* \\ Francisco Herrera Tapia** \\ Cristina Chávez Mejía** \\ Fabiana Sánchez Plata**
}

Resumen: en el presente artículo se presentan los resultados de un estudio del mercado de trabajo rural y su precarización en el municipio de Coatepec Harinas, en el sur del Estado de México, donde se muestra la diversidad ocupacional predominante, así como la especialización en la floricultura, aspectos notables de la nueva ruralidad actual. También, con base en los datos del trabajo de campo, se calculó un índice de precariedad laboral, estratificado en niveles, para saber el grado de precariedad de la población trabajadora.

Palabras clave: mercado de trabajo rural; diversidad ocupacional; población ocupada; precariedad; índice de precariedad; espacio rural.

* Universidad Politécnica del Valle de Toluca. Carretera Toluca-Almoloya de Juárez, km. 5.6, Santiaguito Tlalcilalcali, Almoloya de Juárez, Estado de México, México. C.P. 50904. Teléfono: (722) 400 5385. Correo electrónico: elizagu_80@yahoo.com

** Instituto de Ciencias Agropecuarias y Rurales, Universidad Autónoma del Estado de México. Instituto de Ciencias Agropecuarias y Rurales, campus El Cerrillo, km. 14.5. Carretera Toluca-Atlacomulco s/n, colonia San Cayetano, C. P.: 50295, Toluca, Estado de México, México. Teléfono y fax: (722) 296 5552. Correos electrónicos: fherrerat@uaemex.mx / cchavezm@ uaemex.mx / fsanchezp@uaemex.mx 
Abstract: this article presents the results of a study of the rural labor market and its insecurity in the municipality of Coatepec Harinas, in southern Estado de México. Results show the occupational diversity prevailing in that area, as well as specialization in floriculture, which are notable aspects of the new rurality. Based on the data collected during fieldwork, a Job Security Index was calculated and stratified to show the level of job insecurity among the working population.

Key words: rural labor market; occupational diversity; employed population; job insecurity; Job Security Index; rural space.

\section{Introducción}

El espacio rural ha tenido varios cambios en los últimos años; primero, el incremento de la emigración hacia las zonas urbanas en los años sesenta, producto del crecimiento de las grandes ciudades; segundo, en la década de 1980, con la llegada del modelo neoliberal, el Estado abandonó la política agrícola proteccionista, y los campesinos empezaron a perder apoyos y, por ende, a buscar la diversificación de sus cultivos y, en los años noventa, con la entrada del Tratado de Libre Comercio de América del Norte, su producción agrícola no pudo competir con las grandes industrias agropecuarias de Canadá y Estados Unidos, y se vieron inmersos en la búsqueda de nuevas fuentes de ingresos. Esta situación ha llevado a una recomposición social de las áreas rurales, así como de sus actividades económicas. En la actualidad, más de la mitad de la población se dedica a labores que antes eran exclusivas del espacio urbano, pero que ahora forman parte del quehacer cotidiano de las familias rurales.

En este contexto, los objetivos del presente artículo son analizar la composición y dinámica del mercado de trabajo rural a partir de los cambios en la fuerza laboral y la estructura de las ocupaciones, así como mostrar el cambio sectorial y la calidad de éstas, y utilizar el método de componentes principales para generar un índice de precariedad estratificado en tres niveles: no precario; precario bajo y precario alto, 
con base en datos del Instituto Nacional de Estadística y Geografía (INEGI 2010) y en un estudio de campo donde se aplicó un cuestionario, en 2010, a una muestra de 346 hogares, de un total de 2 047, registrados en el segundo conteo de población de 2005 (INEGI 2010), de las diez localidades principales del municipio de Coatepec Harinas (véase anexo A1), ubicado en el lado sur del Nevado de Toluca, a 35 kilómetros al suroeste de la ciudad de Toluca y a 27 al poniente del de Tenancingo; a 18 al oriente de Texcaltitlán y a 20 al norte de Pilcaya, este último es municipio de Guerrero.

La superficie de Coatepec Harinas es de $280.53 \mathrm{~km}^{2}$, gracias a su ubicación geográfica tiene todo tipo de tierras: riego completo, medio riego, temporal, agostaderos, pastizales, zonas arboladas y también pedregosas. Es un lugar privilegiado por su flora, en donde existe gran diversidad de árboles frutales, un sinnúmero de plantas medicinales, forrajeras y florales. Según datos del INEGI (2010), tiene 36174 habitantes, de los cuales 48 por ciento son hombres y 52 son mujeres.

Coatepec Harinas se caracteriza por cambios visibles en los ámbitos espacial, laboral y social. De tener una amplia vocación para la producción de maíz, pasó a dedicarse a la fruticultura y la floricultura, debido a la crisis económica de 1990 y a las nuevas prácticas traídas por inversores que llegaron para establecer empresas agrícolas. En el año 2000 ocupó el lugar más alto en el índice de intensidad migratoria hacia Estado Unidos, respecto de los demás municipios del Estado de México (Consejo Nacional de Población, CONAPO 2010). A pesar de esto, en el trabajo de campo se encontró que gran parte de su población económicamente activa (PEA) está en situación precaria, aunque esto no es exclusivo del espacio rural, ya que en el urbano ocurre algo similar, como lo demuestran estudios como el de Baca y Castillo (2005).

\section{Mercado de trabajo rural}

Entre los diversos estudios sobre el mercado de trabajo, realizados desde distintos enfoques, destacan los de Bartra (1974) quien, al analizar la estructura agraria del país, se dio cuenta de que los pro- 
ductores del medio rural no son una sociedad homogénea, sino que están divididos en clases, lo que hace notorio un intenso proceso de proletarización del campesinado, imposible de revertir. Por otro lado, Luisa Paré (1977) elaboró un estudio sobre los ingresos de los trabajadores agrícolas, más tarde Astorga (1985) realizó otros sobre el mercado de trabajo rural, al que define como el que implica tanto procesos de producción de peones como la distribución y consumo de fuerza laboral, y se apoya en la teoría de Piore (1983), quien divide al mercado de trabajo en dos grandes segmentos: primario y secundario. En el primero se encuentran los empleos mejor pagados y en el segundo los peor pagados e inestables. Los estudios de Lara (2001) reflejan que la reestructuración de la agricultura mexicana ha profundizado la segmentación del mercado de trabajo rural, lo cual se expresa en las condiciones de contratación y empleo, producto de la flexibilidad laboral; por lo que no se puede dividir sólo en primario y secundario, debido a que en la actualidad se requiere contar con mano de obra capaz, y además la facilidad para contratarla y despedirla en determinado momento.

En México, las investigaciones sobre los mercados de trabajo rurales se han enfocado en los de los asalariados agrícolas, por ejemplo, Barrón (1993) en su tesis doctoral estudió la caracterización del de las hortalizas, Sifuentes (1996) los agrícolas en Nayarit; Muñoz (1997), la mujer jornalera en el valle de Culiacán; Suárez (1997), las michoacanas en el empaque de mangos; Lara (1997), las características del empleo en la floricultura: el caso del Estado de México. Todos dan cuenta de cómo se han modificado dichos mercados desde 1960, a partir de los cambios en el patrón de cultivos, la incorporación de las mujeres, los tipos de ocupaciones que se encuentran y sus condiciones de vida.

El mercado de trabajo rural hacía referencia a las actividades agropecuarias, que le daban el sustento económico a los habitantes del campo. En los años setenta y ochenta, la reproducción social de la clase campesina descansaba en la agricultura (Chayanov 1985), sin embargo en los años noventa, ésta disminuyó como actividad económica principal, y entraron las de los servicios y las industriales, entre otras. Esta diversificación o especialización ocupacional articuló un nuevo mercado de trabajo heterogéneo altamente cambian- 
te, con características diferentes a las de los años previos, con una población más alfabetizada, con acceso a tecnologías y medios de comunicación (Arias 1992). El mercado de trabajo rural forma parte de esos cambios por los que está pasando el espacio rural, que históricamente se definía a partir de dos criterios: ocupacional, relativo a la práctica de actividades agropecuarias, y demográfico, basado en su densidad poblacional (González y Mena 2009). En este estudio se considera al mercado de trabajo rural como el espacio donde se establecen las relaciones productivas y sociales de un lugar (Gutiérrez 1999). Además, se asume que: "Los mercados de trabajo rurales no son sólo oferta y demanda de mano de obra en condiciones segmentadas o excluyentes, sino los lugares en los que se confeccionan las alternativas, entendiéndose por alternativas, la elaboración y consolidación de proyectos multifuncionales que son puestos en valor por los habitantes del territorio" (Torres 2007, 10).

Los cambios en el espacio rural crearon el concepto de ruralidad o rusticidad, debido a las nuevas formas de vida, a la organización particular de sus actividades económicas y a sus nuevos vínculos con la sociedad.

[...] Nueva, por el intento de dar sentido a una experiencia que nos enseña que la ruralidad ha cambiado y por lo tanto debe englobarse en un nuevo concepto y ruralidad, porque permite indicar una experiencia que es aun diferente a lo urbano y en consecuencia no logra prescindir de su contenido histórico [...] (González y Mena 2009, 3).

En el contexto de los cambios globales, los mercados de trabajo rurales se están modificando. La agricultura ha dejado de ser una actividad primaria, para darle paso al surgimiento y consolidación de otras, que han abierto un abanico de ocupaciones en diversos sectores, no exclusivamente los relativos al primario. Los espacios rurales tienen una PEA diferente a la que prevalecía en décadas anteriores, existe más participación femenina, hay mayor grado de escolaridad, acceso a las telecomunicaciones y la movilidad de los mercados regional, nacional e internacional. Estos cambios se ven reflejados en un aumento en la fuerza de trabajo, respecto a la demanda, cuya con- 
secuencia ha sido un deterioro en los salarios y en las condiciones de vida de las familias campesinas, que han incrementado sus actividades económicas, sin embargo, esto sólo se refleja en un aumento en sus ingresos, pero no en sus condiciones laborales cada vez más precarias, carentes de toda prestación y con una jornada laboral amplia.

\section{Precarización del trabajo rural}

México se caracteriza por tener una gran heterogeneidad laboral; hay trabajadores asalariados, que coexisten con los no asalariados (patrones, por cuenta propia, no remunerados); está la gran empresa, con los microestablecimientos; los sectores más modernos de los servicios, con los vinculados a la economía de subsistencia, y los empleos formales, que conviven con los informales (De Oliveira 2006). En este mercado, donde están inmersos todo tipo de trabajadores, y donde la persistencia y profundización de la heterogeneidad en la estructura productiva sigue siendo un poderoso elemento explicativo de la precariedad laboral en el México actual (Mora y De Oliveira 2009). Se entiende como precariedad laboral al conjunto de condiciones en que operan los trabajadores en lo que concierne a la actividad en la que se desempeñan: a) la discontinuidad en el tiempo; b) la inexistencia de contratos; c) la falta de respeto al salario mínimo; d) los horarios muy amplios y e) la carencia de seguridad social, entre otros aspectos, que sólo tienen consecuencias negativas (Arriagada 1994, citado en Baca y Castillo 2005).

Para Brígida García (2006), el principal problema que enfrenta la población activa del país no es la falta absoluta de ocupaciones, sino de empleos con remuneraciones adecuadas y otras condiciones satisfactorias. La autora identificó los siguientes ejes analíticos con respecto a un mercado laboral precario: a) ampliación de la insuficiente absorción laboral; b) importancia del trabajo no asalariado ligado a la subsistencia o a la pobreza; c) permanencia de las bajas retribuciones; d) inestabilidad laboral frecuente; e) inseguridad en constante aumento (mayor presencia de trabajadores temporales con contratos transitorios o sin ellos); f) declive de la cobertura de protección social; g) menor importancia de la negociación colectiva y h) pérdidas 
en el ámbito de los derechos laborales $(2006,38)$. Estas características son una muestra de las debilidades en el funcionamiento de las instituciones y de la falta de atención por parte de los agentes económicos y de los actores político-institucionales, para regular las condiciones socioeconómicas de la población ocupada (PO).

Los ejes analíticos que señala García (2006) se confirman de manera especial en el contexto rural, como el que aquí se describirá, ya que se trata de territorios expulsores de migrantes, lo que denota la absorción laboral insuficiente del municipio de estudio, donde prevalece un esquema flexible y no asalariado, con retribuciones bajas e inestabilidad laboral y desconocimiento de los derechos laborales. Hechos y condiciones a los que las campesinas se han ido adaptando a través de diversas estrategias, como la diversificación de ingresos y la emigración a Estados Unidos o a las zonas metropolitanas de México.

Las políticas neoliberales en los estados y la apertura a la competencia internacional han ocasionado el deterioro general de las condiciones laborales de los asalariados, además de las pocas oportunidades del propio empleo asalariado provocando la propagación de estrategias de supervivencia, como la informalidad. Es decir, se ha polarizado la economía, y ha aumentado la precarización entre los trabajadores, sobre todo crece la vulnerabilidad de las mujeres y se limitan más sus oportunidades de acceso a empleos con las condiciones laborales mínimas que marca la ley (Pedrero 2009).

Mercedes Pedrero afirma que "[...] el mercado ya es plenamente flexible y presiona sobre el mercado formal y da lugar a la caída del salario $[\ldots] "(2009,120)$ además, también se ve reflejado en la jornada, en las prestaciones y en que se realiza más de una actividad, que no necesariamente es formal. Pedrero realizó este estudio en el ámbito urbano, por lo que en el espacio rural esta situación es más marcada, puesto que siempre ha carecido de prestaciones y salarios dignos. Además de que existe precariedad no sólo en el mercado de trabajo, sino también en las instituciones gubernamentales que deberían regular las actividades económicas.

A partir de la década de 1990 se agudizaron los cambios en materia laboral, que en la actualidad prevalecen en el campo, como la incorporación de la mujer en ocupaciones remuneradas y la mayor 
participación de la Po en diversas actividades económicas, lo que dejó de lado la preocupación por la reproducción de los esquemas tradicionales de subsistencia, como lo eran las labores agrícolas. Algunos habitantes emigran en busca de alternativas económicas, que les permitan una movilidad social, y otros se quedan. Sin duda, todos estos cambios traen efectos sobre el mercado local y global de trabajo.

Otro acercamiento a la precariedad laboral es el de los nuevos trabajos, que expresan la terciarización creciente de la economía y el surgimiento de formas inéditas de organización (De la Garza 2010). Como se muestra más adelante en esta investigación, se encontró que en el espacio rural se tiende a incrementar la participación de la PO en el sector terciario, con la finalidad de diversificar su ingreso, debido también a que la actividad agropecuaria dejó de ser rentable para muchos de los y las productoras rurales.

\section{Composición y dinámica del mercado de trabajo rural en el municipio de Coatepec Harinas, Estado de México}

En los últimos años, la PEA ${ }^{1}$ se ha incrementado de forma considerable, debido a cambios demográficos y a la creciente incursión de la mujer al mercado de trabajo; en la actualidad representa más de la mitad de la población total del municipio donde, una tercera parte está constituida por mujeres, cuya participación ha aumentado a más del doble. También ha disminuido la PO en la agricultura, para incorporarse al sector servicios, sin embargo, esta situación no ha ocasionado una mejora en el nivel de vida de los habitantes de Coatepec Harinas, al contrario, sus ingresos son precarios -una buena parte de la población recibe cuando mucho dos salarios mínimos- (INEGI

\footnotetext{
${ }^{1}$ La PEA incluye a todas las personas de ambos sexos que constituyen la mano de obra disponible para la producción de bienes y servicios (entre 12 y 64 años), y que declararon realizar o buscar una actividad económica. Dentro de ella se distingue la Po, que es la que trabaja por lo menos una hora o un día, para producir bienes o servicios de manera independiente o subordinada, con o sin remuneración, y los desocupados, quienes carecen de empleo, pero lo buscan o están a la expectativa de alguno. La población económicamente inactiva es la que no tiene una actividad económica ni la busca, puede tratarse de inactivos, de pasivos transitorios (jóvenes) o de pasivos definitivos (ancianos); entre los inactivos se incluye a las amas de casa y a los religiosos, entre otros (INEGI 2010).
} 
2010), por lo que algunos de ellos, para aumentar sus ingresos, tienen más de una ocupación que, en muchas ocasiones, no les ofrece prestaciones, lo que genera la expansión de formas precarias de contratación y uso de la fuerza de trabajo (Castillo 2001).

En 1970, Coatepec Harinas tenía 45 personas económicamente activas por cada 100 habitantes de 12 años y más, la tasa de participación económica más alta se daba entre los de 45 a 49 años. Datos del censo de 2010 muestran que dichas personas son 50 por cada 100 habitantes, de las cuales la tasa de participación más alta está entre el mismo rango de edad que en 1970. Estos datos indican que, a pesar de que a escala nacional la tasa de participación económica se concentra en población más joven (30-34 años), en Coatepec Harinas no ocurre lo mismo, esto producto de su alta emigración, por lo que su población joven se encuentra laborando en otros países, principalmente en Estados Unidos, lo que deja en su comunidad a niños, mujeres y adultos.

En lo referente a la ocupación por sectores, en Coatepec Harinas destaca que el primario es el más fuerte, sin embargo, su participación ha ido disminuyendo, en relación con la del industrial, que se ha mantenido relativamente constante, aunque es el terciario el que más ha crecido a lo largo del tiempo, como se observa en la figura 1.

De 1970 a 2010, en lo que respecta a la distribución de la PO por sector, se ha visto ese cambio del agropecuario al industrial y luego al de servicios, y también un incremento considerable en la participación de mujeres y hombres en el comercio, lo que muestra que en el municipio de estudio se cuenta con una diversidad económica, rasgo característico asociado a una nueva ruralidad.

La estructura ocupacional se ha ido modificando en las últimas décadas, la agricultura, por ejemplo, ha trasferido mano de obra a los sectores secundario y terciario, en forma considerable. En general, la localización de las actividades industriales, comerciales y de servicios ha ocasionado la migración interna y la inmigración de grupos que también estaban ocupados o subocupados en otras regiones del Estado de México, por lo que han cambiado radicalmente su actividad económica. En 1970, 74 por ciento de la PEA se ubicaba en el sector primario, mientras que en 2010 , el porcentaje era de 56 , con un incremento importante al de servicios, lo que muestra un gran 
cambio en la situación ocupacional de los habitantes de Coatepec Harinas.

\section{Figura 1}

Población ocupada, por sector en Coatepec Harinas, Estado de México, 1970-2010 (porcentaje)

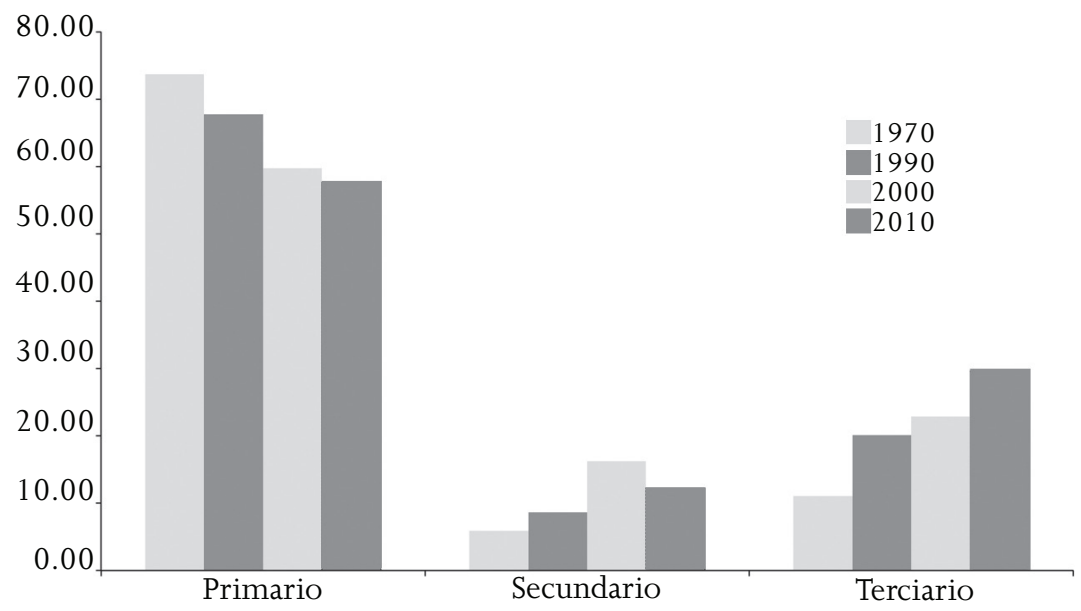

Fuente: INEGI $(2010,2000,1990,1970)$.

Según datos del INEGI $(2010,2000,1990,1970)$, que consideran la distribución de las distintas ocupaciones de la población de Coatepec Harinas, de 1970 a 2010, en 1970 predominaban la de obrero y del trabajador en labores agropecuarias, en ese periodo, el comercio todavía no tenía mucho auge.

Para 1990, la estructura ocupacional cambió, y se incorporaron los trabajadores de la educación, del arte, los operadores de maquinaria fija, de trasporte, los ambulantes, los domésticos, de protección y vigilancia, y se separó el rubro de profesionistas y técnicos; predominó la ocupación en las labores agropecuarias, la de artesano y obrero, seguido de la de comerciante, también se sumaron más, referentes al sector servicios. 
En el año 2000 hubo un descenso de trabajadores agropecuarios y un incremento en artesanos, obreros y comerciantes. Datos del INEGI (2010) muestran un municipio diversificado, donde más de 32 por ciento de su población se dedicaba a actividades completamente diferentes a las agropecuarias (ídem.), por lo que las nuevas decisiones gubernamentales deben tomar en consideración el cambio laboral que experimenta el espacio rural, y no sólo crear programas en apoyo al campo sino a todos los sectores económicos que hoy prevalecen en la zona. Es decir, la implementación de políticas públicas integrales con un fuerte componente intersectorial, que logre la articulación de las actividades productivas del territorio.

\section{Condiciones de las ocupaciones productivas en Coatepec Harinas, Estado de México}

La buena o mala calidad de las ocupaciones corresponde a si son asalariadas y no asalariadas, caracterizadas por la baja calidad, la inestabilidad en el empleo y, en particular, por la escasa seguridad en los ingresos (Baca y Castillo 2005, 312). La calidad no está en función de realizar una actividad formal o informal, como ya se mencionó, sino que puede ocurrir en los dos ámbitos. Como señala Castillo (2001), la precariedad del trabajo se delimita en términos de las relaciones, formas o tipos de vinculación laboral y no de un sector adscrito, es decir, lo que normalmente se vincula al informal.

A continuación se muestran datos obtenidos de la aplicación del cuestionario en 2010 en el municipio de Coatepec Harinas, con lo cual se pretende mostrar las condiciones laborales de su PEA. A pesar de que ya se contaba con datos del censo 2010, la información era insuficiente para las expectativas de la investigación, puesto que no toda estaba estratificada, además el trabajo de campo permitió tener un mayor acercamiento y observar de forma directa las condiciones de precariedad prevalecientes en el lugar de estudio.

En la figura 2 aparece la distribución de la PO en Coatepec Harinas; la mayoría se dedica a labores relativas a los sectores agropecuario y terciario, lo que muestra la existencia de un mercado de trabajo rural diversificado. 


\section{Figura 2}

Población ocupada, por trabajo principal en Coatepec Harinas, Estado de México, 2010 (porcentaje)

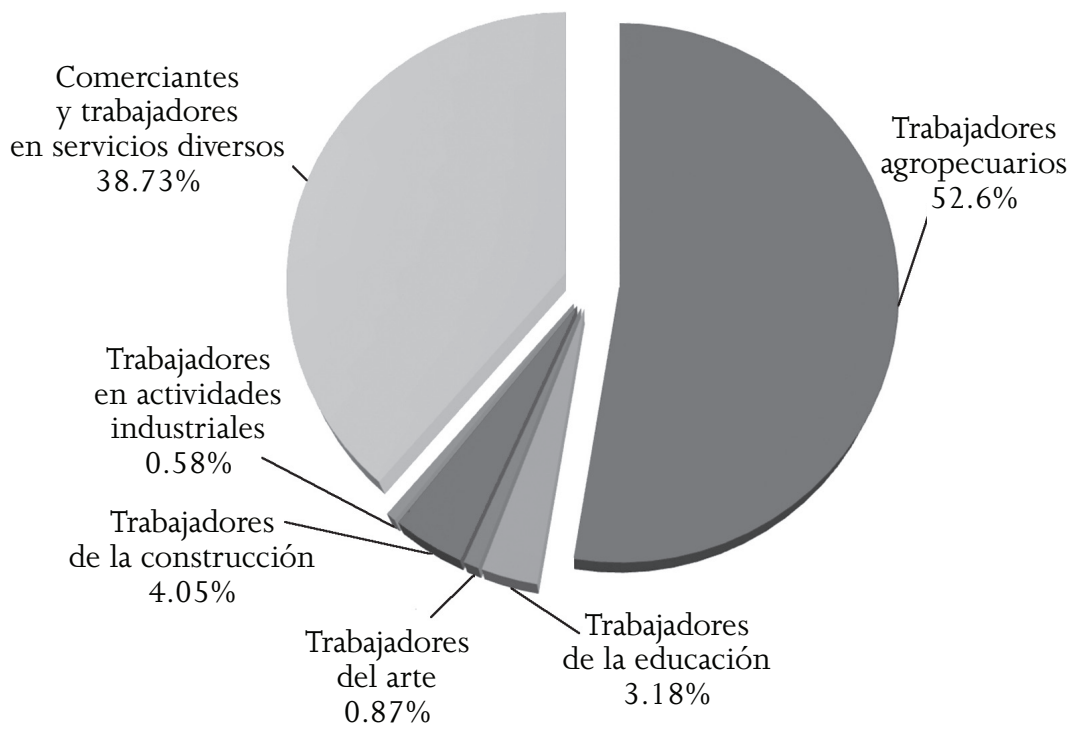

Fuente: elaboración propia, con base en los resultados del trabajo de campo ( $\mathrm{n}=346)$.

Respecto a su condición de ocupación, 53 por ciento de la población a la que se le aplicó el cuestionario trabajaba por su cuenta (véase figura 3). Esta situación pudiera parecer favorecedora, pero no se refleja en el mejoramiento de sus ingresos ni en sus condiciones laborales, ya que la mayoría tiene pequeños negocios o se dedica a labores del campo, sin que existan mayores opciones de crecimiento debido a la precariedad económica de la zona donde ofrece sus productos o servicios al público. 20 por ciento de los encuestados eran asalariados, localizados en empresas dedicadas a la floricultura, cuyas condiciones laborales también eran precarias, lo que confirma que la precariedad no está adscrita al sector informal, sino que ocurre en ambos. 


\section{Figura 3}

Población ocupada, por situación en el trabajo en Coatepec Harinas, Estado de México, 2010 (porcentaje)

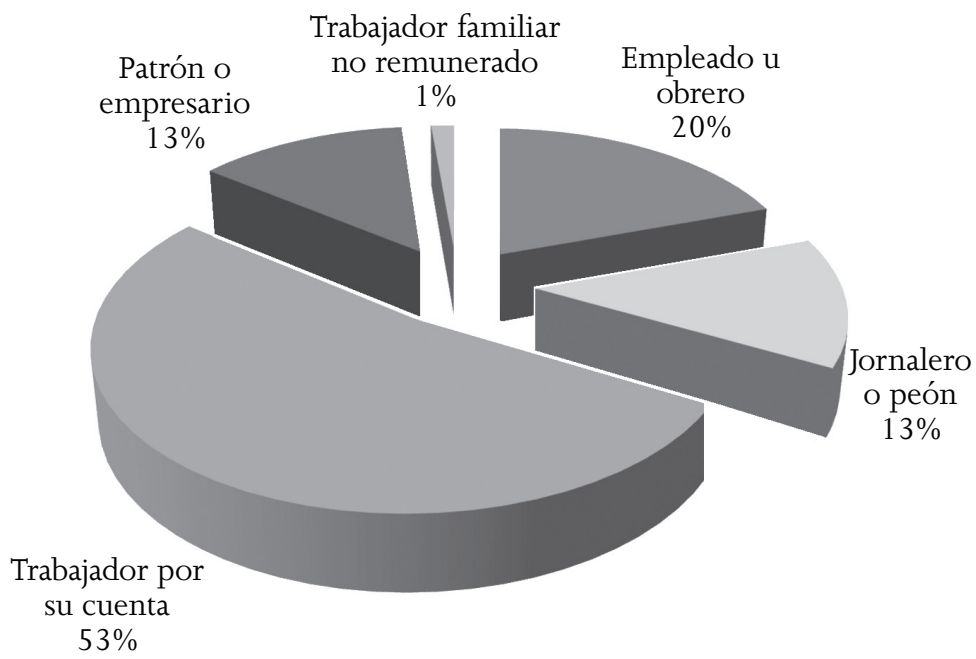

Fuente: elaboración propia, con base en los resultados del trabajo de campo (n=346).

En la figura 4 se muestran las condiciones laborales de los encuestados, en lo referente a seguridad social, ingreso y jornada, con base en su situación en el trabajo. Como se puede constatar, en el caso de los empleados u obreros, menos de la mitad de ellos contaba con contrato (29 por ciento) y tenía alguna prestación (29), pero los jornaleros o peones no tenían prestaciones laborales y para ambos, así como para los trabajadores por cuenta propia, ${ }^{2}$ para los patrones $^{3}$ o los empresarios, más de la mitad tenía una jornada laboral

\footnotetext{
${ }^{2}$ Persona ocupada que desempeña su oficio o profesión, sólo o asociado con otros, no tiene trabajadores remunerados a su cargo, pero puede disponer de algunos sin pago (Secretaría del Trabajo y Previsión Social, STPS 2012).

${ }^{3}$ Es el trabajador independiente que emplea personas a cambio de una remuneración económica en dinero (ídem.).
} 


\section{Figura 4}

Condiciones laborales de la población ocupada por situación en el trabajo, Coatepec Harinas, Estado de México, 2010 (porcentaje)

\begin{tabular}{|c|c|c|c|c|c|}
\hline $\begin{array}{c}\text { Situación en } \\
\text { el trabajo }\end{array}$ & $\begin{array}{c}\text { Empleado } \\
\text { u obrero }\end{array}$ & $\begin{array}{c}\text { Jornalero } \\
\text { o peón }\end{array}$ & $\begin{array}{c}\text { Trabajador } \\
\text { por su } \\
\text { cuenta }\end{array}$ & $\begin{array}{c}\text { Patrón o } \\
\text { empresario }\end{array}$ & $\begin{array}{l}\text { Trabajador } \\
\text { familiar no } \\
\text { remunerado }\end{array}$ \\
\hline Total & 20 & 13 & 53 & 13 & 1 \\
\hline Tienen empleados & - & - & $7^{*}$ & 100 & - \\
\hline $\begin{array}{l}\text { Pertenecen a } \\
\text { un sindicato }\end{array}$ & 19 & 0 & - & - & - \\
\hline Tienen contrato & 29 & 0 & - & - & - \\
\hline Tienen prestaciones & 29 & 0 & - & - & - \\
\hline $\begin{array}{c}\text { Tienen seguridad } \\
\text { social }\end{array}$ & 31 & 0 & - & - & - \\
\hline $\begin{array}{l}\text { Jornada laboral } \\
\text { + de } 40 \text { hrs }\end{array}$ & 68 & 80 & 68 & 78 & 0 \\
\hline $\begin{array}{l}\text { Ingreso semanal } \\
\text { menor a } \$ 1000\end{array}$ & 63 & 92 & 86 & 76 & - \\
\hline
\end{tabular}

Fuente: elaboración propia, con base en los resultados del trabajo de campo $(n=346)$.

* Trabajadores no remunerados.

superior a las 40 horas semanales y, en general, más de 63 por ciento de esta PEA percibía menos de mil pesos semanales.

Debido a la falta de prestaciones laborales, a la baja calidad de las ocupaciones, a la inestabilidad en el empleo, a la escasa seguridad social y a los bajos ingresos, se puede afirmar que las condiciones de trabajo de esta PEA son precarias y, pese a que se ha movido a otros sectores, no ha logrado mejorar su situación laboral, por lo que en algunos casos tiene más de un empleo.

Hasta este momento se han mostrado las condiciones laborales de la población de estudio, que no están tan alejadas de las de otras comunidades rurales de todo el país, donde, según datos de la Encuesta nacional de ocupación y empleo (ENOE 2010), del INEGI, en el tercer trimestre de 2010, 57 por ciento de la PO ganaba entre dos y tres salarios mínimos. 


\section{Índice de precariedad laboral para Coatepec Harinas, Estado de México}

García (2006), De Oliveira (2006), Guadarrama et al. (2012) y el European Study of Precarious Employment (ESOPE 2004) analizaron la precariedad desde un enfoque cualitativo, mediante estudios etnográficos, considerando condiciones laborales y niveles salariales, y coincidieron en que aspectos como la inestabilidad laboral, la poca cobertura de la protección social, el incremento del trabajo no asalariado y los bajos salarios se han vuelto estructurales y debilitan el carácter integrador del trabajo en las sociedades contemporáneas. Son pocos los que utilizan un enfoque cuantitativo, como Castillo (2001), quien realizó un estudio en Panamá, donde analizó las tendencias de precarización, y construyó un índice en el cual consideró el salario mínimo, la jornada laboral, a los trabajadores por cuenta propia y a los patrones de establecimientos con menos de cinco empleados. Considera que la precariedad del trabajo se delimita en términos de las relaciones, formas o tipos de vinculación laboral y no de un sector adscrito. Mora (2010) construyó un índice de precariedad y lo dividió en tres niveles: no precario, precario bajo y precario alto, para lo que tomó en cuenta la estabilidad y la jornada laboral, la remuneración salarial y la seguridad social.

A pesar de que son diversas las investigaciones que han calculado los índices de precariedad, se han focalizado en el espacio urbano, donde predomina el trabajo asalariado. Hoy en día, en el medio rural predominan patrones de consumo, hábitos de vida y tantas actividades económicas como en la ciudad donde, a pesar de que se desempeñaban labores pertenecientes al sector primario, cuyo trabajo se realizaba principalmente por cuenta propia, en la actualidad hay mucha población asalariada en ese sector (Lara 2001; INEGI 2010). En el contexto de la nueva ruralidad, la creciente movilidad de la población ha modificado el patrón de organización del territorio, y desplazado casi por completo las fronteras entre lo rural y urbano (Carton de Grammont 2004), por lo que se puede construir un índice de precariedad para el espacio rural, cuyas características y actividades económicas son similares, debido a la interacción existente entre los dos espacios. 
El índice de precariedad para Coatepec Harinas se basa en la clasificación de Mora (2010), debido a que el estudio es de tipo transversal, no se puede hacer un análisis de tendencia, además la información que se tiene de fuentes secundarias es insuficiente. En ese sentido, con base en los datos obtenidos en el trabajo de campo, se analizó el grado de precariedad de la Po de Coatepec Harinas, en 2010. Esta medida de precarización del trabajo se elaboró con base en los indicadores de mala calidad del empleo, encontrados a lo largo de esta investigación en el mercado de trabajo que se estudia (Castillo 2001; Mora y De Oliveira 2009), y en otras sobre este tema (véase figura 5).

\section{Figura 5}

Indicadores de precariedad laboral

\begin{tabular}{|c|c|c|}
\hline Variable & Descripción & Indicador \\
\hline Ingreso & $\begin{array}{l}\text { El empleo precario es el que reporta } \\
\text { menos de dos salarios mínimos }\end{array}$ & $\begin{array}{c}\text { Salario mínimo (sal_min) } \\
\text { 0: dos o más salarios mínimos } \\
\text { 1: menos de dos salarios mínimos }\end{array}$ \\
\hline $\begin{array}{c}\text { Situación } \\
\text { en el } \\
\text { trabajo }\end{array}$ & $\begin{array}{c}\text { Está conformada por lo ocupados } \\
\text { que trabajan en forma independiente, } \\
\text { que no tienen patrón ni empleados } \\
\text { remunerados a su cargo }\end{array}$ & \begin{tabular}{|c|} 
Trabajadores por cuenta \\
propia (trab_cta) \\
0: no trabajador por cuenta propia \\
1: trabajador por cuenta propia
\end{tabular} \\
\hline $\begin{array}{c}\text { Situación } \\
\text { en el } \\
\text { trabajo }\end{array}$ & $\begin{array}{c}\text { Personas que poseen algún } \\
\text { establecimiento, y que disponen } \\
\text { de algún trabajador remunerado en } \\
\text { dinero o especie }\end{array}$ & $\begin{array}{c}\text { Patrones de establecimientos con } \\
\text { menos de cinco empleados } \\
\text { (patr_mcinco) } \\
\text { 0: no patrones o patrones } \\
\text { con cinco o más } \\
\text { 1: patrón con menos de cinco }\end{array}$ \\
\hline $\begin{array}{l}\text { Jornada } \\
\text { laboral }\end{array}$ & $\begin{array}{l}\text { La completa cuando es de } \\
35 \text { a } 48 \text { horas semanales }\end{array}$ & $\begin{array}{c}\text { Horas trabajadas (jornada_comple) } \\
\text { 0: de } 35 \text { a } 48 \text { hrs } \\
\text { 1: menos de } 35 \text { o más de } 48 \text { hrs }\end{array}$ \\
\hline $\begin{array}{l}\text { Seguridad } \\
\text { social }\end{array}$ & $\begin{array}{c}\text { Identifica a los trabajadores que } \\
\text { tienen acceso a instituciones de salud }\end{array}$ & $\begin{array}{c}\text { Acceso o no a las instituciones } \\
\text { de salud (seg_soc) } \\
\text { 0: tiene seguridad social } \\
\text { 1: no tiene seguridad social }\end{array}$ \\
\hline Contrato & $\begin{array}{c}\text { El empleo precario es cuando los } \\
\text { trabajadores están sujetos a procesos } \\
\text { de contratación en un plazo definido } \\
\text { o laboran sin contrato }\end{array}$ & $\begin{array}{l}\text { Tipo de contrato (contrato) } \\
0: \text { cuenta con uno escrito } \\
\text { 1: no cuenta con uno escrito }\end{array}$ \\
\hline
\end{tabular}

Fuente: elaboración propia, con base en Castillo (2001) y Mora y De Oliveira (2009). 
Para calcular este índice se hace un análisis de factores utilizando el método de componentes principales (Ávila 2007), que es una técnica de reducción de datos, que sirve para encontrar grupos homogéneos de variables a partir de un conjunto de éstas; los grupos se forman con las variables donde existe una alta correlación. El valor del índice varía en sentido directo al grado de precarización; a mayor valor del índice, más desfavorable es la situación ocupacional o viceversa.

El análisis de factores consistió en cuatro fases, en la primera se calculó una matriz capaz de mostrar la variabilidad conjunta de todas las variables: la comunalidad de una variable es la proporción de su varianza, que se puede explicar por el modelo factorial obtenido, las variables trabajador por su cuenta y patrón con menos de cinco empleados son las mejor explicadas. El modelo es capaz de reproducir 98 por ciento de su variabilidad original (véase anexo A2). En la segunda se obtuvo la extracción de número óptimo de factores, el procedimiento extrae dos que consiguen explicar 69.5 por ciento de la varianza de los datos originales, lo que se considera como un buen análisis de factores (véase anexo A3).

La tercera fase muestra la rotación de la solución original, la cual indica que el primer factor está constituido por las variables: trabajador por su cuenta y patrón con menos de cinco empleados, y parece reflejar la dimensión de situación en el trabajo; el segundo factor está formado por la variable salario mínimo, jornada semanal, seguridad social y contrato, lo cual representa la estabilidad laboral (véase anexo A4), y la última consistió en hacer el cálculo de índice de precariedad combinando cada factor obtenido por la variabilidad explicada por cada uno de ellos.

\section{Estratificación de índice}

Una vez obtenido el índice de precariedad, que fue de 0.7936, se estratifica para diferenciar a la población de Coatepec Harinas, según niveles de precariedad de acuerdo con sus características laborales. Para estratificar una sola variable, que en este caso es el índice de precariedad laboral, se utilizó el criterio de estratificación de mínima 
varianza, obtenida por Dalenius (1950, citado por Mora 2010). Este método consiste en hallar una estratificación óptima, definida como aquélla en la cual la varianza del estimador sea mínima dentro de los grupos (estratos), es decir, lograr que éstos sean lo más homogéneo posible en su interior y lo más heterogéneo posible y diferenciables entre sí. La variable resultante de estratificación, expresada en puntajes, es continua y tiene una distribución aproximadamente normal, lo que garantiza la partición óptima de estratos.

Con el análisis de factores se estimó el índice de precariedad laboral, se estratificó en tres niveles, mostrados en la figura 6, donde se observa que 79 por ciento de la población se encuentra en el precario alto, lo que implica ingresos inferiores a dos salarios mínimos, jornadas extensas, trabajar sin contrato y sin acceso a servicio médico, o bien, si trabajan por su cuenta, sus negocios son pequeños, por lo que la mayoría cuenta con menos de cinco empleados. Sólo 6.6 por ciento de la población se encuentra en condiciones no precarias, y en su mayoría desempeña puestos en la educación, es trabajador público y uno que otro es empresario, con negocios en expansión de más de cinco trabajadores.

\section{Figura 6}

Niveles de precariedad laboral de la población de Coatepec Harinas, Estado de México, 2010

\begin{tabular}{|c|c|c|}
\hline Nivel & Absolutos & Relativos (\%) \\
\hline No precario & 23 & 6.6 \\
\hline Precario bajo & 50 & 14.4 \\
\hline Precario alto & 273 & 79 \\
\hline Total & 346 & 100 \\
\hline
\end{tabular}

Fuente: elaboración propia, con base en los resultados de trabajo de campo $(n=346)$.

\section{Conclusiones}

En este artículo se mostraron los cambios en el mercado de trabajo, como el incremento en la participación económica de la mujer, la 
variedad de labores agropecuarias, por ejemplo en Coatepec Harinas se incursionó en las frutícolas y florícolas, y se produjo un cambio sectorial, ahora el espacio rural está diversificado, en cuanto a sus principales actividades económicas, pues son muy similares a las que prevalecen en los espacios urbanos.

En la actualidad, en el municipio de Coatepec Harinas, así como en el estado y el país, más de 50 por ciento de la población está en edad de trabajar, en lo que respecta al lugar de estudio, la tasa de participación económica masculina es de 79 por ciento, mientras que la femenina es de 23, por lo que existe una gran población circulando en el mercado laboral.

En la estratificación del índice de precariedad se muestra claramente que más de la mitad de la población vive en condiciones precarias, es decir, gana menos de dos salarios mínimos, su jornada es amplia y si tiene un negocio es precario, ya que no cuenta con posibilidades de crecimiento por falta de capital y asesoría técnica empresarial para brindar mejores condiciones laborales a sus escasos trabajadores.

Por lo anterior, se requiere una reforma laboral encaminada a mejorar la calidad de las ocupaciones. Y, para el espacio rural, crear programas que impulsen la productividad y competitividad, que tomen en cuenta la heterogeneidad de ocupaciones que en él prevalecen. También se justifica el diseño y puesta en marcha de políticas integrales con un enfoque intersectorial, que vayan acompañadas de una social de amplia cobertura, para que se logre reconocer la creciente diversificación de las actividades productivas y a la par fomentar estrategias tendientes a evitar los desequilibrios regionales, la inequidad y la precariedad laboral existentes en las relaciones entre el capital y el trabajo.

A quienes toman las decisiones en materia de políticas públicas, enfocadas al desarrollo regional rural de fomento a la productividad, se les sugiere que generen incentivos para elevar la calidad del empleo y ampliar la cobertura de los sistemas de protección social; también que establezcan acciones que tomen en cuenta la variedad de actividades económicas prevalecientes, así como el tipo de población a las que van dirigidas, que en su mayoría son mujeres y jóvenes quienes habitan en los espacios rurales, para que no emigren a los 
mercados internacionales, sino que hagan de su lugar de origen uno próspero y productivo, que mejore las condiciones socioeconómicas de todos sus habitantes.

Recibido en marzo de 2014 Aceptado en septiembre de 2014

\section{Bibliografía}

Arias, Patricia. 1992. Nueva rusticidad mexicana. México: Consejo Nacional para la Cultura y las Artes.

Astorga, Enrique. 1985. Mercado de trabajo rural en México. México: Editorial Colección Problemas de México.

Ávila, Carmelo. 2007. Análisis factorial. Curso del doctorado estadística multivariante aplicada. http://biplot.usal.es/DOCTORADO/3CiCLO/ BIENIO-06-08/MetodosClasicos/AFactorial.pdf

Baca, Norma y Dídimo Castillo. 2005. Precarización ocupacional por género en la zona metropolitana de la ciudad de Toluca. Convergencia 37: 289-326.

Barrón, María Antonieta.1993. Los mercados de trabajo rurales: el caso de las hortalizas en México. Tesis de doctorado, Facultad de Economía, Universidad Nacional Autónoma de México (UNAM).

Bartra, Roger. 1974. Estructura agraria y clases sociales en México. México: Editorial ERA.

Carton de Grammont, Hubert. 2004. La nueva ruralidad en América Latina. Revista Mexicana de Sociología 66 (número especial): 279-300.

Castillo, Dídimo. 2001. Los nuevos precarios, ¿mujeres u hombres? Tendencias en el mercado de trabajo urbano en Panamá, 19821999. Papeles de Población 27: 99-145. 
Chayanov, Alexander. 1985. La organización de la unidad económica campesina. Buenos Aires: Editorial Nueva Visión.

CONAPO. 2010. Índices de intensidad migratoria, México Estados Unidos (2000). http://www.conapo.gob.mx/index.php?option= com_content\&view=article\&id=317\&Itemid=15 (Página $(27$ de noviembre de 2010).

Devore, Jay. 2001. Probabilidad y estadística para ingeniería y ciencias. México: Editorial Thomson.

ENOE. 2010. Encuesta nacional de ocupación y empleo, tercer trimestre de 2010: INEGI.

ESOPE. 2004. A comparative study of labor market related risk in flexible economies. Informe final. Bruselas: Comisión Europea de Investigación.

García, Brígida. 2006. La situación laboral precaria: marcos conceptuales y ejes analíticos pertinentes. Trabajo 2 (3): 23-52.

Garza, Enrique de la. 2010. Trabajo a-típico, iidentidad o fragmentación?: alternativas de análisis. En Trabajos atípicos y precarización del empleo, coordinado por Edith Pacheco, Enrique de la Garza y Luis Reygadas, 49-76. México: El Colegio de México (COlmex).

González López, Felipe y Rodrigo Mena. 2009. Lo rural como construcción sociopolítica. Revista Latinoamericana de Desarrollo Humano. Chile, boletín 57 .

Guadarrama, Rocío, Alfredo Hualde y Silvia López. 2012. Precariedad laboral y heterogeneidad ocupacional: una propuesta teóricometodológica. Revista Mexicana de Sociología (2): 213-243.

Gutiérrez, Estela. 1999. Nuevos escenarios en el mercado de trabajo en México, 1983-1999. Papeles de Población (21): 21-55.

INEGI. 2010. Censo general de población y vivienda. 
2005. II Conteo de población.

2000. XII Censo general de población. Estado de México, México.

. 1990. XI Censo general de población. Estado de México, México.

. 1970. IX Censo general de población. Estado de México, México.

Lara, Sara María. 2001. Análisis del mercado de trabajo rural en México en un contexto de flexibilización. En ¿Una nueva ruralidad en América Latina?, 363-380. Buenos Aires: Consejo Latinoamericano de Ciencias Sociales.

. 1997. El mercado de trabajo en la floricultura de exportación. El caso del Estado de México. En Mercados de trabajo rurales en México, coordinado por Antonieta Barrón y Lorena Sifuentes, 181192. México: UNAM.

Lipschutz, Seymour. 1999. Introducción a la probabilidad y estadística. Madrid: Editorial Mc Graw Hill.

Mora, Minor. 2010. Ajuste y empleo: la precarización del trabajo asalariado en la era de la globalización. México: COLMEX.

y Orlandina de Oliveira. 2009. La degradación del empleo asalariado en los albores del siglo xxI: Costa Rica y México. Papeles de Población 15 (61): 195-231.

Muñoz, Amparo. 1997. La mujer jornalera del valle de Culiacán, Sinaloa, un estudio de caso. En Mercados de trabajo rurales en México, coordinado por Antonieta Barrón y Lorena Sifuentes, 141-179. México: COLMEX.

Oliveira, Orlandina de. 2006. Jóvenes y precariedad laboral en México. Papeles de Población 12 (49): 37-73. 
Paré, Luisa. 1997. El proletariado agrícola en México. ¿Obreros agrícolas o campesinos sin tierra? México: Siglo XXI.

Pedrero, Mercedes. 2009. Las condiciones de trabajo a principios del siglo Xxi. Presencia de las mujeres en el sector informal. Papeles de Población 15 (59): 119-171.

Piore, Michael. 1983. Notas para una teoría de la estratificación del mercado de trabajo. En El mercado de trabajo:teorías y aplicaciones, compilado por Luis Toharia, 193-223. Madrid: Alianza.

Sifuentes, Lorena. 1996. Los mercados de fuerza de trabajo en la agricultura de Nayarit en el periodo 1970-1994 y la participación femenina. Tesis de maestría. Facultad de Economía, UNAM.

STPS. 2012. Secretaría del Trabajo y Previsión Social: http://www. stps.gob.mx/bp/secciones/conoce/areas_atencion/web/Glosario/glosario.htm\#t (15 de abril de 2012).

Suárez, Blanca. 1997. Las mujeres michoacanas en el empaque de mangos. En Mercados de trabajo rurales en México, coordinado por Antonieta Barrón y Lorena Sifuentes, 121-139. México: UNAM.

Torres, Gerardo. 2007. Agroindustria rural y mercados de trabajo. ¿Alternativa a la pobreza rural? Cuadernos PROLAM/USP VII (2). 


\section{Anexos}

A1

Tamaño de la muestra de las localidades seleccionadas para el trabajo de campo

\begin{tabular}{|c|c|c|}
\hline Localidad & Número de hogares & Tamaño de la muestra \\
\hline $\begin{array}{c}\text { Coatepec Harinas } \\
\text { (cabecera municipal) }\end{array}$ & 1315 & 135 \\
\hline Acuitlapilco & 336 & 34 \\
\hline Chiltepec de Hidalgo & 222 & 23 \\
\hline Llano Grande & 292 & 30 \\
\hline Meyuca & 192 & 20 \\
\hline San José & 212 & 22 \\
\hline Tecolotepec & 155 & 16 \\
\hline Las Vueltas & 205 & 21 \\
\hline Primera de Analco & 270 & 17 \\
\hline Piedras Anchas & 163 & 346 \\
\hline Total de hogares & 2047 & \\
\hline
\end{tabular}

Fuente: elaboración propia, con base en la fórmula para el cálculo de la muestra (Lipschutz 1999).

Para calcular la muestra se utilizó la siguiente fórmula:

$$
n=\frac{N p q}{\left[\frac{M e^{2}}{N c^{2}}(N-1)\right]+p q}
$$

donde:

$\mathrm{n}=$ tamaño de la muestra

$\mathrm{N}=$ tamaño del universo

$\mathrm{p}=$ probabilidad de ocurrencia: 0.5

$\mathrm{q}=$ probabilidad de no ocurrencia: 0.5

Me $=$ margen de error o precisión: $+/-5 \%$ de margen de error $(0,05)$

$\mathrm{Nc}=$ nivel de confianza o exactitud, expresado con valor de $z$, que determina el área de probabilidad buscada. 95\% de nivel de confianza, expresado como valor de z: 1.96. 


\section{A2}

Comunalidades, 2010

\begin{tabular}{|c|c|c|}
\hline & Inicial & Extracción \\
\hline Salario mínimo & 1.000 & 0.467 \\
\hline Trabajador por su cuenta & 1.000 & 0.981 \\
\hline Patrón con menos de cinco trabajadores & 1.000 & 0.981 \\
\hline Jornada semanal completa & 1.000 & 0.020 \\
\hline Seguridad social & 1.000 & 0.869 \\
\hline Contrato & 1.000 & 0.855 \\
\hline
\end{tabular}

Método de extracción: análisis de componentes principales.

\section{A3}

Factores óptimos: total de varianza explicada, 2010

\begin{tabular}{|c|c|c|c|c|c|c|c|c|c|}
\hline \multirow{2}{*}{ Componente } & \multicolumn{3}{|c|}{ Autovalores iniciales } & \multicolumn{3}{|c|}{$\begin{array}{l}\text { Sumas de las saturaciones al } \\
\text { cuadrado de la extracción }\end{array}$} & \multicolumn{3}{|c|}{$\begin{array}{l}\text { Suma de las saturaciones } \\
\text { al cuadrado de la rotación }\end{array}$} \\
\hline & Total & $\begin{array}{l}\% \text { de la } \\
\text { varianza }\end{array}$ & \begin{tabular}{c|}
$\%$ \\
acumulado
\end{tabular} & Total & $\begin{array}{c}\% \text { de la } \\
\text { varianza }\end{array}$ & $\begin{array}{c}\% \\
\text { acumulado }\end{array}$ & Total & $\begin{array}{c}\% \text { de la } \\
\text { varianza }\end{array}$ & $\begin{array}{c}\% \\
\text { acumulado }\end{array}$ \\
\hline 1 & 2.261 & 37.690 & 37.690 & 2.261 & 37.690 & 37.690 & 2.095 & 34.919 & 34.919 \\
\hline 2 & 1.912 & 31.860 & 69.550 & 1.912 & 31.860 & 69.550 & 2.078 & 34.631 & 69.550 \\
\hline 3 & .991 & 16.513 & 86.063 & & & & & & \\
\hline 4 & .698 & 11.626 & 97.689 & & & & & & \\
\hline 5 & .139 & 2.311 & 100.000 & & & & & & \\
\hline 6 & $-4.090 \mathrm{E}-17$ & $-6.817 \mathrm{E}-16$ & 100.000 & & & & & & \\
\hline
\end{tabular}

Método de extracción: análisis de componentes principales.

A4

Estructura de factores rotados, 2010

Matriz de componentes rotados ${ }^{\mathrm{a}}$

\begin{tabular}{|c|c|c|}
\hline \multirow{2}{*}{} & \multicolumn{2}{|c|}{ Componente } \\
\cline { 2 - 3 } & 1 & 2 \\
\hline Salario mínimo & -0.223 & 0.646 \\
\hline Trabajador por su cuenta & 0.990 & -0.019 \\
\hline Patrón con menos de cinco trabajadores & 0.990 & -0.019 \\
\hline Jornada semanal completa & -0.063 & 0.128 \\
\hline Seguridad social & 0.199 & 0.911 \\
\hline Contrato & 0.202 & 0.902 \\
\hline
\end{tabular}

Método de extracción: análisis de componentes principales. Método de rotación: normalización varimax con Kaiser.

a. La rotación convergió en tres iteraciones. 
